\title{
Contraceptive Knowledge, Beliefs and Attitudes in Rural Malawi: Misinformation, Misbeliefs and Misperceptions
}

\section{Effie K. Chipeta ${ }^{1}$, Wanangwa Chimwaza ${ }^{1}$, Linda Kalilani-Phiri ${ }^{1}$}

1.Center for Reproductive Health, College of Medicine, University of Malawi

Abstract

\section{Introduction}

The objective of the study was to find out factors that affect the intentions of men and women to use family planning methods.

Methods

The study was conducted in Mangochi district, the southern region of Malawi in 2006.

About 60 Focus group discussions were conducted to identify the attitudes and beliefs of 30 male and 30 female participants regarding family planning methods. The data was then transcribed and analyzed manually identifying central themes and relationships across the cases and narratives.

\section{Results}

Most of the respondents knew the different types of family planning methods and reported that health facilities had adequate stock of family planning supplies. However, it was found that most of the women and men were not using any modern family planning methods. Reasons included: family planning methods were perceived side effects, such as prolonged menstruation, men's concerns about impotence and genital sores, weight gain or loss, and subsequent infertility. Traditional family planning methods were mostly used for infertility problems.

\section{Conclusion}

Despite knowing about the different types of family planning methods, and awareness of their ready availability in health facilities, use of these methods is low because considerable misinformation still prevails regarding contraceptive methods' side effects.

\section{Introduction}

Family planning reduces the number of unintended and unwanted pregnancies and thereby saving women from high risk pregnancies and unsafe abortions ${ }^{1}$. Other benefits accruing from family planning methods include prevention of cancers, sexually transmitted infections (STIs) and infection with the Human Immunodeficiency Virus (HIV) ${ }^{2}$. Furthermore, investing in family planning as a component of good reproductive health has benefits that go beyond the obvious prevention of pregnancy and reduction of disease burden, the social and economic benefits for global development goals should not be overlooked ${ }^{3}$.

However, despite persistent advocacy urging the use of modern contraceptive methods for family planning, the fertility rates in most sub-Saharan African countries still remains unacceptably high ${ }^{4}$, mostly due to poor uptake of contraception because of cultural, economical and political barriers $^{5}$. Like most developing countries Malawi's total fertility rate (TFR) still remains high at 6.3 births per woman ${ }^{6}$. It has been estimated that about half of the total demand for contraception in Malawi remains unmet and, consequently, approximately $22 \%$ of pregnancies are unwanted ${ }^{7}$.
In an attempt to improve family planning uptake in Malawi, a project called Promoting Sexual and Reproductive Health in Malawi (PSRHM) was introduced in Lungwena and Makanjira area in Mangochi district located in the southern region of Malawi. The project is conducted jointly by the government of Malawi through the Ministry of Health and the College of Medicine. In this paper, we report on the data that was collected during this project on the knowledge, attitudes and behaviour of people towards modern family planning methods.

\section{Methods}

The study took place in Lungwena and Makanjira area in Mangochi district, which is located in the southern region of Malawi in 2006. The project was approved by the College of Medicine Research and Ethics Committee (COMREC) prior to conducting the study. The data was collected using focus group discussions (FGDs) with members of the community on perceptions about sexual and reproductive health (SRH) service delivery at the health facilities in the study areas.

\section{Focus Group Discussions}

FGDs were conducted in both Makanjira and Lungwena. Participants for these FGDs were selected based on gender and age. A total of 60 FGDs, each with 8-10 participants, were conducted: 20 with adolescents (15-19 years), 20 with young adults (20-34 years) and 20 with adults (35-65 years) resident in 10 sampled villages ( 5 from Lungwena and 5 from Makanjira). The FGDs were done in Yao, the local language and were recorded on an audio tape. A trained facilitator led each discussion while another person took notes which were later were translated in English. The FGDs addressed the various topics including attitudes and perceptions towards SRH services and factors influencing use of modern contraceptives.

\section{Results}

\section{Background Characteristics of the FGDs Participants}

Including all the participants in the FGDs and Exit Interviews, a total of 1,115 people ( 899 women and 216 men) took part in this study. The median age for men was 34 years (range $12-78$ years) and the median age for the women was 26 years (range 12-74 years).

Most the participants were married (men $(79.2 \%$ and women $83.7 \%$ ). The majority of respondents belonged to Yao tribe $76 \%(\mathrm{n}=847)$, with $7.8 \%(\mathrm{n}=87)$ and $6 \%(\mathrm{n}=67)$ of the

people being Chewa and Lomwe respectively. Most of the respondents were Moslems 76.1\% ( $n=847)$ with the remainder being Catholics 5.7\% $(\mathrm{n}=64)$ and Protestants $14.1 \%(\mathrm{n}=157)$. More men had attended school $76.1 \%$ $(\mathrm{n}=164)$ compared to females $63.8 \%(\mathrm{n}=574)$.

\section{Knowledge on Family Planning Policies and Programs}

The majority of the participants said that the Malawi government encourages child birth spacing, use of modern 
family planning methods and has education programs on

problems of overpopulation. Additionally, participants said that government policy discourages early child bearing by youth, and that government advocates the use of family planning methods by all women within the reproductive age group. Both male and female respondents identified the radio as the major source of information.

"We mostly hear on radio that we should be going to hospitals to collect family planning pills, condoms and other contraceptive methods." (Female participant)

Concerning government programmes or strategies, the respondents said that the government makes sure that contraceptives like pills, condoms, loop, insertions and other methods are available to the public and are easily accessible. Most of the participants reported that the health facilities have adequate stocks of family planning supplies. They also indicated that government encourages family planning through awareness raising activities such as community drama and radio programs. They further mentioned that the distribution of contraceptives through the door to door approach using Community-Based Distribution Agents (CBDAs) is one of the government's programmes that is aimed at increasing access of the family planning methods, because the CBDAs agents go from door to door informing and counselling both married and unmarried individuals on family planning and distributing pills and condoms in the villages.

Both male and female respondents recommended the hospital as the best place to access family planning services compared to using the CBDAs because the hospital offers a wide range of family planning methods such as oral contraceptives, condoms, the loop, Norplant, injectables, tubal ligation, and spermicides, whereas CBDAs only distribute oral contraceptives and condoms. The respondents also said they prefer the hospital because it is more private and workers are more confidential than CBDAs.

\section{Use of Modern Contraceptives}

We found that the majority of women were not using any method of contraceptive to prevent or postpone pregnancy. The major reasons for not using family planning methods included lack of knowledge and partner refusal.

"Our husbands tell us that if one is married and according to the Muslim faith, she should expect to have more children". (Female participant)

Among women who reported using modern contraception, Depo-Provera (injectable) was the most commonly reported family planning method used. The women reported that they prefer this method because it is easy to hide the use from their partners.

Injection is on great demand because it is secretive. Your husband will not know that you are using any contraceptive method'. (Female participant).

Although oral contraceptive pills are also popular, most women do not like using them because they hear health workers telling them that pills cause damage to organs.

"When we get sick because of using pills, at the hospital we are told that the pills have clotted in the intestines and in the uterus and the organs are rotting". (Female participant)

Several men also reported not using any family planning method. For men who were using family planning methods, the condom was the most reported method.

\section{Traditional versus Facility Based Family Planning Methods}

The participants reported that only a few people use traditional family planning methods unlike in the past when they were used widely.

"But it is not always hospital, others go for local African medicine and it helps". (Male participant)

The respondents also mentioned that traditional methods are mostly used by older women.

"Those who use traditional methods are women with several children not for young girls. It does not work in girls because they are more fertile". (Female participant)

Of the traditional methods reported, two were most frequently mentioned-- watery medicine and the string method. The majority of the respondents said that most people go to a traditional healer for infertility problems and very few report to the hospital.

"Many go to the African doctors and others go to the hospital to become fertile". (Male participant)

According to most female respondents, the function of traditional healers is to restore fertility.

"For problems with infertility, we visit herbalists". (Female participant)

Some respondents said that they have never heard of infertility problems being sorted out at the hospital, they strongly believe that traditional healers are the only experts in infertility issues.

"At the hospital, no doctor has announced that they deal with such problems". (Female participant)

There is a belief that Ngokota (pain in the body and lower limbs during menstruation) is due to witchcraft and that it renders a woman barren. This has further strengthen the practice of going to traditional healers for infertility problems.

"It is better to go to traditional healer because after getting herbs and eat, you find the pain has stopped and after 2-3 months a woman gets pregnant. The herbs really belp a lot". (Female participant)

\section{Attitudes towards Modern Contraceptives}

Although the respondents reported that family planning services are available in their areas, we found that some women do not use the modern family planning methods because of the negative attitudes, myths and beliefs that surround the use of family planning methods. We found that there was a common belief among female respondents that contraceptives affect male reproductive organs, causing men to be impotent.

"If a man sleeps with a woman who is taking contraceptives, its power renders him weak.(impotent)". (Female participant)

Male respondents also reported that condoms cause blisters and sores in their genitalia while others felt that condoms have an oily substance which kills their manhood and

weakens their sexual strength " there is an oily substance produced in condoms which causes sores in private parts and this weakens our manhood" (Male participant)

Furthermore, men also expressed concern over some of the methods which their wives use. Some men indicated that the foam produced by foam tablets weakens their manhood.

Additionally, most married men indicated that they are irritated with the prolonged menstruation which usually occurs in women using Depoprovera, because less time is 
available for sex.

"Depo-Provera (injection), is not allowing us to enjoy marriage life because our wives are just menstruating without stopping" (Male participant)

Some of the female respondents indicated that they do not use family planning methods because of fear of side effects like prolonged menstruation, heart palpitations and obesity.

Others reported that contraceptives make people to lose weight and they feel ashamed as people suspect them of having HIV/AIDS.

Despite the fact that most women and men had indicated negative attitude towards using family planning methods as highlighted above, some respondents had a positive attitude towards them. The main reasons given in support of using modern family planning methods included, having the desired number of children, lack of financial support for the children, experiencing pregnancy or delivery complications in previous pregnancies, desire to maintain good maternal health and participate in development work and family problems like unfaithfulness of a partner. Additionally, in situations where men have several partners, condoms were identified to be useful because they help prevent disease transmission from one partner to the other.

"Condoms are not for family planning but are for sex. They are good to use with extramarital partners". (Male participant)

\section{Decision Making and Communication about Family Planning Methods}

Most of the women reported that whilst they saw the importance of using modern family planning methods, using these methods depended mostly on husband's approval. Most women indicated they could not use family planning because their husbands wanted more children and therefore disapproved the use of family planning methods.

"If one is married, she should expect to have more children". (Female participant)

The female respondents, who indicated that they discuss issues of family planning with their partners, mentioned that they are the ones who initiate discussion on family planning issues They also indicated that most men in their area are not interested in family planning; therefore the women take it as their responsibility to encourage their partners because women are the ones who experience the pain of child bearing.

"It is us women who start discussing this issue of family planning with our husband because it is a woman who bears the pain when they are pregnant". (Female participant)

Women who have never given birth or only have one child indicated that they do not discuss family planning issues with husband simply because they feel that they are not ready for family planning, and from their experience with other women they have seen that family planning prevents women from becoming pregnant at the moment they want to have children. Other women indicated that they are scared to discuss family planning issues with their spouse because they fear their spouses could beat them. Others felt that there is no need to discuss family planning issues with a man simply because 'this family planning is a woman thing'.

Most men reported that discussing family planning with their partners is a waste of time simply because they are against the use of family planning methods. The men claimed that the use of family planning causes a lot of problems to most women. The younger men reported that they are not ready for family planning and therefore they can't talk about family planning with their spouses.

\section{Discussion}

We found that among the focus group participants knowledge of modern family planning method is high in the study area. In addition the majority of participants reported modern family planning methods are available at the nearest health facility with most women and men reporting stocks of contraceptives to be adequate. Furthermore, respondents reported that a variety of family planning methods are available at health facilities including oral contraceptive pills, injectables, condoms, insertions, the loop, tubal ligation and spermicides. However despite the fact that knowledge of modern family planning methods is high and that these methods are readily available in the study area, more than half of the women and men reported not using any of these methods. This finding is similar to results found in several other studies conducted in Malawi and elsewhere ${ }^{8-11}$. This implies that knowledge and availability of the modern family planning methods alone does not determine use of these services and that other factors influence decisions on whether or not to adopt a modern family planning methods.

Respondents reported that main motivating factors for using modern family planning methods were having the desired number of children, lack of finances to support children,

experiencing pregnancy or delivery complications in previous pregnancies, the desire to maintain good maternal health and participate in development work, and family problems. These findings indicated that most respondents were aware of the benefits of modern family planning. This result is comparable to the findings of another study that was conducted in Nigeria $^{2}$ where they found that improved standard of living, children's education, strength and beauty of the mother were the main factors encouraging the use of modern family planning methods.

One of the main factors that discouraged women from using modern family planning methods in our study was husband's disapproval. In several other studies, husband's approval was also found to be a determinant of women's intention to use modern family planning methods ${ }^{2,12-14}$. This may be because of the authority that men have over household decisions and the high regard that wives have of their husbands which is the case in most cultures. Most of the respondents in this study also mentioned fear of side effects as a factor influencing their intention to use modern family planning methods.

However looking at the responses, not all the side effects that were mentioned are related to contraceptives. Nonetheless, other studies have found fear of side effects to be one of

the most important for non use of modern family planning methods ${ }^{15,17}$.

Apart from modern family planning methods, we also found that some women use traditional family planning methods. Unlike other studies where the most commonly reported traditional methods were the withdrawal method and periodic abstinence ${ }^{18,20}$, two forms of traditional family planning methods were reported in this study; watery medicine which is drunk as a concoction and a string which is worn around a woman's waist and is believed to prevent pregnancy. We also found that only few people still use traditional method for family planning except for infertility reasons.

Additionally, respondents indicated these traditional methods 
are used by older women who are less likely to get pregnant. This result indicates that people prefer modern family planning methods probably because they are more effective than traditional methods.

This is similar to the results that were obtained in the Malawi Demographic Health Survey (MDHS) were the use of traditional family planning method dropped from $43 \%$ in 1992 to $15 \%$ in $2000^{6}$.

\section{Conclusion}

These findings demonstrate that people's intentions to use modern family planning methods are affected by perceived benefits, concerns about how side effects may influence their daily lives and personal assessment of how particular methods may affect relationships with partners. Thus in order for family planning intervention programs to be more effective there is need to include continuous counselling and education to help people distinguish fact from fiction regarding health and side effects of the modern family planning methods. There is also need to encourage more male participation in reproductive health activities so as increase their support to women on these matters.

\section{References}

1. White J, Speizer I. Can family planning outreach bridge the urbanrural divide in Zambia? BMC Health Serv Res.2007;7:143

2. Moronkola O, Ojedirin M, Amosu A, Reproductive health knowledge, Beliefs and determinants of use among women attending Family planning clinics in Ibadan, Nigeria. Afric Health Sci 2006, 6:155-159

3. MacPhail C, Pettifor A, et al, Contraception use and pregnancy among 15-24 year old South African women: a nationally representative crosssectional survey. BMC medicine 2007, 5:31

4. Audu B, Yahya J, Bassi A. Knowledge, Attitude and practice of natural Family planning methods in a population with poor utilization of modern Contraceptives. Obs \& Gyn 2006;26:555-560.

5. Konje J, Ladipo O. Barriers to uptake and use of modern methods of contraception in developing countries, International Journal of Gynecology \& Obstetrics 1999 287-294

6. National Statistical Office. Malawi National Demographic and Health Survey 2000. 2000 National Statistical Office, Zomba.

7. Ministry of Health- Government of Malawi. Reproductive Health Policy. 2002.

8. Bisika T, Kasote A, et al. Avoiding Unwanted Pregnancy and Sexually Transmitted Infections: A Rural Malawi District Study, Chapel Hill, NC: Measure Evaluation; 2004

9. Munthali A.C, Chimbiri A, Zulu E. Adolescent Sexual Reproductive Health In Malawi: A Synthesis of Research Evidence. 2004

10. Orji E.O and Onwudiegwu, Prevalence and Determinants of Contraceptive Practice in a defined Nigerian Population. Journal of Obstetrics and Gynaecology. 2000 ; 22(5):540-543

11. Obisesan K.A, Adeyemomo A.A and Fakokunde B.O, Awareness and use of Family Planning Methods among Women in Ibadan, Nigeria. East African Journal Medical Journal.1998; 75:135-138

12. El-zanaty, Fatma, Way A, Kishor S. and Casterline J. Indepth Study on the Reasons for Non-Use of Family Planning- Cairo: National Population Council. 1999

13. Mishra, Vinod K, Robert D, Retherford, Nair P.S and Griffith F. Reasons for Discontinuing and Not Intending to Use Contraception in India. National Family Health Survey Reports No 13. Mumbai India: International Institute of Population Science 1999

14. Stash, Sharon. Explanations of Unmet Need for Contraception in Chitwan, Nepal. Stud Fam Plan 1999;30(4):267-287
15. Campbell M, Hodoglugil N, Potts M, Barriers to Fertility Regulation: A review of the Literature, Stud Fam Plan 2006; 32: 87-98

16. Casterline J. Sathar Z, Haquel M, Obstacles to Contraceptive Use in Pakistan : A study in Punjab, Stud Fam Plan 2001;32:95-110

17. Sedgh G. Bankole et al, Unwanted Pregnancy and Associated Factors Among Nigerian Women. Int Fam Plan Per 2006;32:175-184

18. Althaus F, Malawi's Fertility Declines, Infant and Child Mortality Rates Remain Elevated, Int Fam Plan Per. 1994; 20(4):162-164

19. Bledsoe C.H. Hill A.G et al. Constructing Natural Fertility: The Use of Western Contraceptive Technologies in Rural Gambia. Population and Development Review 1994;20:81-113

20. Ekani-Bessala M. Carre N. Prevalence and Determinants of Contraceptive Method Use in a Palm Oil Company in Cameroon. Contraception 1998; 58:29-3 